\title{
In situ stress determination from inversion of hydraulic fracturing data
}

\author{
Jinsong Huang \\ Research Associate Professor, Division of Engineering, Colorado School of Mines, Golden, CO 80401 \\ (corresponding author). E-mail:jhuang@mines.edu, Tel:1-303-273-3173, Fax:1-303-273-3602
}

D. V. Griffiths

Professor, Division of Engineering, Colorado School of Mines, Golden, CO 80401. E-mail:

d.v.griffiths@mines.edu

\section{Sau-Wai Wong}

Shell International Exploration and Production, Email: sau-wai.wong@shell.com

\begin{abstract}
The paper presents a new method based on rigorous principles of mechanics, for determining the in-situ rock stress state based on hydraulic fracturing data. A solution can be obtained from a single data set which includes breakdown pressure, fracture angular position and trace angle. The inversion methodology is demonstrated on a case history from the Kuparuk River field, Alaska as reported by Pearson et al. (1992), and shown to give good agreement with observed field data.
\end{abstract}

Keywords: Hydraulic fracture, in situ stress, inversion, inclined wellbores

\section{Introduction}

Hydraulic fracturing is the best-known method of assessing the state of in situ stress at great depths. The technique originated in the 1940s as an oilfield stimulation technique designed to intensify production by fracturing a segment of a wellbore through pressurization; the fracture was then extended by additional pumping and maintained open (or propped) by injecting solid particles such as sand grains or glass beads. Following the great success of hydraulic fracturing as a stimulation technique, attempts were made to understand the mechanisms behind it. The most important effort in the interpretation of hydraulic fracturing mechanism was made by Hubbert and Willis (1957) who used the theory of elasticity to reach the conclusion that the direction of the induced hydraulic fracture and the pressures recorded during borehole pressurization are 
directly related to the principal in situ stresses. Fairhurst (1964) was among the first to advocate the use of hydraulic fracturing for in situ stress determination. Haimson and Fairhurst (1967) extended Biot's theory of poro-elasticity to include both nonpenetrating and penetrating injection fluid cases. Hydraulic fracturing has now become one of the key methods for in-situ rock stress estimation as suggested by the International Society for Rock Mechanics (ISRM) (Haimson and Cornet 2003). A detailed history of the method and a thorough description of the equipment, setup, test data interpretation and in situ stress derivation are presented by Haimson (1993).

Although hydraulic fracturing is commonly conducted in vertical holes, testing of inclined or deviated wellbores is often required. For example, in the petroleum industry inclined holes are increasingly used for added efficiency in production. In geotechnical site investigations exploratory inclined holes are often drilled in order to intersect vertical joint sets which could be missed by vertical holes. Additionally, rock properties or drilling difficulties may cause unintended deviated holes.

A technique for inverting results from a minimum of two leak-off tests at different well inclinations and azimuths was presented by Aadnøy (1990a, 1990b). This method gives an estimate of both horizontal stress magnitudes and directions. However, the published technique suffers from the assumption that shear stresses are neglected. Gjønnes et al. (1998) present results based on a model which also includes the shear stresses. Djurhuus and Aadnøy (2003) presens a theory for determining the in situ stress state from multiple fracturing data and induced fractures from image logs. A solution can be obtained with a minimum of two data sets. However, using an inversion technique, a solution can be obtained with any number of data sets, as the solution is over determined.

A fundamental understanding of fracture initiation in arbitrarily inclined wellbores under various in situ initial stress conditions is essential for the efficient and effective design of hydraulic fracture systems. The basic equations describing the stress distribution around a horizontal, vertical and inclined wellbore may be derived from the solutions developed by Kirsch (1898), Fairhurst (1968) and Bradley (1979) respectively. It is general believed that a fracture initiates when the maximum tensile stress induced at any point around the wellbore exceeds the tensile strength of the formation at that point. When this occurs, the resulting fracture on the wellbore wall will have an orientation that is perpendicular to the direction of the most tensile principal stress. The angle between the wellbore generatrix and the fracture orientation on the wellbore wall is called the trace angle (e.g., Daneshy 1971, Kuriyagawa et al., 1989, Peška and Zoback 1995, Aadnøy and Bell 1998) which can be observed by high-resolution electrical imaging technologies (e.g., Barton et al., 1997). Some previous attempts have been made to use the fracture trace angle to determine in situ stresses (e.g. Peška and Zoback 1995, Djurhuus and Aadnøy 2003). This paper presents a new method for determining the in situ stress state from hydraulic fracturing data. It is shown that an analytical solution can be obtained when fracture trace angle is available. Later in the paper, this new method is applied to data from the Kuparuk River field in Alaska as reported by Pearson et al. (1992), and shown to predict in-situ stresses in good agreement with field data. When fracture trace angles are not available, other incomplete inversion methods can be used, such as "leak-off" data from the North Sea (e.g. Djurhuus and Aadnøy, 2003 and Gjønnes et al. 1998). 


\section{Theoretical development for stresses and hydraulic fracturing}

Hydraulic fracturing consists of sealing off a short segment (typically $0.5-2 \mathrm{~m}$ ) of a wellbore or borehole at the desired depth, injecting fluid (usually water) into it at a rate sufficient to raise the hydraulic pressure quite rapidly (typically $0.1-1.0 \mathrm{MPa} / \mathrm{s}$ ), and bringing about hydraulic fracturing. The latter is achieved when the borehole fluid pressure reaches a critical level called breakdown pressure. At breakdown the rock fractures in tension causing borehole fluid loss and hence a drop in pressure. When pumping is stopped, the hydraulic line to the testing interval remains in place. Following fracture, the pressure immediately decays, at first very quickly as the fluid chases the still extending fracturing tip, and then more slowly as the fracture closes, after which the only remaining fluid loss is due to seepage into the rock through the borehole wall. The "shut-in pressure" occurs at the transition between the fast and slow pressure decay and signifies the closure of the fracture.

The in situ principal stresses are assumed to be vertical and horizontal. The rock is assumed to be isotropic, homogeneous and linearly elastic. The ambient pore pressure in the rock is assumed to remain constant during the test. However, the method presented can easily be extended to cases where fluid penetrates into surrounding rock, in which case poro-elastic theory must be adopted.

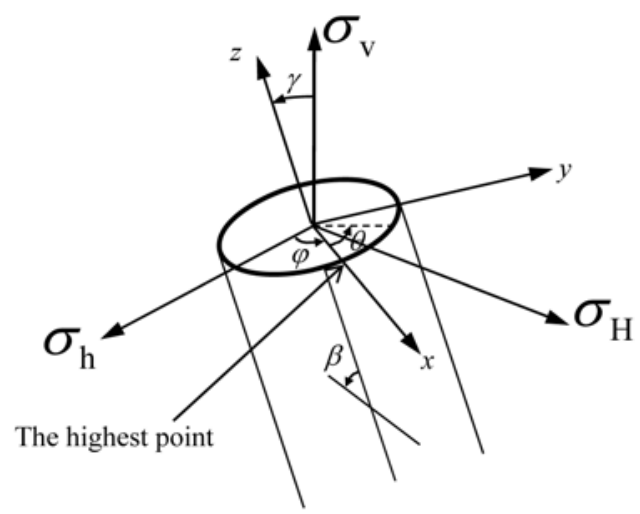

Fig. 1 Wellbore configuration

Referring to Fig. 1 and assuming a compression positive convention, let $\sigma_{\mathrm{v}}, \sigma_{\mathrm{H}}$ and $\sigma_{\mathrm{h}}$ $\left(\sigma_{\mathrm{H}}>\sigma_{\mathrm{h}}\right)$ be the initial in situ vertical and horizontal principal stresses ( $\sigma_{\mathrm{H}}$ is the most compressive horizontal principal stress). Let the origin of the principal stress axes lie at the center of the top of the inclined wellbore shown in the figure. Consider the local coordinates of the wellbore $(x, y, z)$ with the same origin, where the $x$-axis passes through the highest point of the circumference and the $z$-axis passes down the longitudinal axis. Two angular rotations are needed to describe the orientation of the wellbore relative to the in situ principal stress directions:

1. The wellbore azimuth $\varphi$ is the horizontal angle between the vertical plane containing the $\sigma_{\mathrm{h}}$-axis and the vertical plane containing the $x$-axis, measured counterclockwise as viewed down the $\sigma_{\mathrm{v}}-$ axis looking towards the origin. 
2. The wellbore inclination $\gamma$ is the angle between the $\sigma_{\mathrm{v}}$-axis and the $z$-axis measured clockwise as viewed down the $y$-axis looking towards the origin.

The geometry of fractures initiated along an arbitrarily inclined wellbore is strongly dependent on in situ stresses $\left(\sigma_{\mathrm{v}}, \sigma_{\mathrm{H}}\right.$ and $\left.\sigma_{\mathrm{h}}\right)$, wellbore azimuth $(\varphi)$ and inclination $(\gamma)$. The fracture trace angle $\beta$ between the fracture trace and wellbore generatrix is measured in a clockwise direction when looking outwards from the wellbore axis.

For an arbitrarily oriented wellbore, the rotation of the stress tensor from the in situ coordinate system to a local wellbore coordinate system (Fig. 1) is given by

$$
\left\{\begin{array}{c}
\sigma_{x} \\
\sigma_{y} \\
\sigma_{z} \\
\tau_{x y} \\
\tau_{y z} \\
\tau_{z x}
\end{array}\right\}=\left[\begin{array}{ccc}
\cos ^{2} \varphi \cos ^{2} \gamma & \sin ^{2} \varphi \cos ^{2} \gamma & \sin ^{2} \gamma \\
\sin ^{2} \varphi & \cos ^{2} \varphi & 0 \\
\cos ^{2} \varphi \sin ^{2} \gamma & \sin ^{2} \varphi \sin ^{2} \gamma & \cos ^{2} \gamma \\
-\sin \varphi \cos \varphi \cos \gamma & \sin \varphi \cos \varphi \cos \gamma & 0 \\
\sin \varphi \cos \varphi \sin \gamma & -\sin \varphi \cos \varphi \sin \gamma & 0 \\
-\cos ^{2} \varphi \sin \gamma \cos \gamma & -\sin ^{2} \varphi \sin \gamma \cos \gamma & \sin \gamma \cos \gamma
\end{array}\right]\left\{\begin{array}{c}
\sigma_{\mathrm{h}} \\
\sigma_{\mathrm{H}} \\
\sigma_{\mathrm{v}}
\end{array}\right\}
$$

Let $\theta$ be the angle measured counterclockwise starting at $\theta=0$ on the $x$-axis as viewed down the $z$ - axis looking towards the origin. The stress solutions in cylindrical coordinates $(r, \zeta, \theta)$, around the wellbore are given as (e.g. Hiramastu and Oka 1968; Fairhurst 1968):

$$
\begin{aligned}
\sigma_{r}= & \left(\frac{\sigma_{x}+\sigma_{y}}{2}\right)\left(1-\frac{a^{2}}{r^{2}}\right)+\left(\frac{\sigma_{x}-\sigma_{y}}{2}\right)\left(1+3 \frac{a^{4}}{r^{4}}-4 \frac{a^{2}}{r^{2}}\right) \cos (2 \theta) \\
& +\tau_{x y}\left(1+3 \frac{a^{4}}{r^{4}}-4 \frac{a^{2}}{r^{2}}\right) \sin (2 \theta)+P_{w} \frac{a^{2}}{r^{2}} \\
\sigma_{\theta}= & \left(\frac{\sigma_{x}+\sigma_{y}}{2}\right)\left(1+\frac{a^{2}}{r^{2}}\right)-\left(\frac{\sigma_{x}-\sigma_{y}}{2}\right)\left(1+3 \frac{a^{4}}{r^{4}}\right) \cos (2 \theta) \\
& +\tau_{x y}\left(1+3 \frac{a^{4}}{r^{4}}\right) \sin (2 \theta)-P_{w} \frac{a^{2}}{r^{2}} \\
\sigma_{\zeta}= & \sigma_{z}-v\left[2\left(\sigma_{x}+\sigma_{y}\right) \frac{a^{2}}{r^{2}} \cos (2 \theta)+4 \tau_{x y} \frac{a^{2}}{r^{2}} \sin (2 \theta)\right] \\
\tau_{r \theta}= & \left(\frac{\sigma_{x}-\sigma_{y}}{2}\right)\left(1-3 \frac{a^{4}}{r^{4}}+2 \frac{a^{2}}{r^{2}}\right) \sin (2 \theta)+\tau_{x y}\left(1-3 \frac{a^{4}}{r^{4}}+2 \frac{a^{2}}{r^{2}}\right) \cos (2 \theta)
\end{aligned}
$$




$$
\begin{gathered}
\tau_{\theta \zeta}=\left(-\tau_{x z} \sin \theta+\tau_{y z} \cos \theta\right)\left(1+\frac{a^{2}}{r^{2}}\right) \\
\tau_{r \zeta}=\left(\tau_{x z} \cos \theta+\tau_{y z} \sin \theta\right)\left(1+\frac{a^{2}}{r^{2}}\right)
\end{gathered}
$$

where $a$ is the wellbore radius, $P_{w}$ is the compressive applied wellbore pressure, $r$ is the radial distance from the wellbore center, and $v$ is Poisson 's ratio.

It can be seen from Eq. (2) that fracture initiated from the wellbore reorients itself once it extends beyond the zone of the wellbore influence and becomes normal to the least compressive in situ principal stress (e.g., Daneshy 1971, Chen and Economides 1999).

Considering stresses at the wellbore wall $(r=a)$

$\sigma_{r}=P_{w}$

$\sigma_{\theta}=\sigma_{x}+\sigma_{y}-2\left(\sigma_{x}-\sigma_{y}\right) \cos (2 \theta)-4 \tau_{x y} \sin (2 \theta)-P_{w}$

$\sigma_{\zeta}=\sigma_{z}-v\left\{2\left(\sigma_{x}-\sigma_{y}\right) \cos (2 \theta)+4 \tau_{x y} \sin (2 \theta)\right\}$

$\tau_{r \theta}=0$

$\tau_{\theta \zeta}=-2 \tau_{x z} \sin \theta+2 \tau_{y z} \cos \theta$

$\tau_{\zeta r}=0$

thus the effective stress tensor is

$$
\sigma^{\prime}=\left[\begin{array}{ccc}
\sigma_{r}^{\prime} & 0 & 0 \\
0 & \sigma_{\theta}^{\prime} & \tau_{\theta \zeta} \\
0 & \tau_{\theta \zeta} & \sigma_{\zeta}^{\prime}
\end{array}\right]
$$

where $\sigma_{r}^{\prime}=\sigma_{r}-P_{0}, \sigma_{\theta}^{\prime}=\sigma_{\theta}-P_{0}, \sigma_{\zeta}^{\prime}=\sigma_{\zeta}-P_{0}$, and $P_{0}$ is the pore pressure.

The effective principal stresses at the wellbore can be found as the eigenvalues of the effective stress tensor, thus

$$
\sigma_{1}^{\prime}=\sigma_{r}^{\prime}
$$


$\sigma_{2}^{\prime}=\frac{\left(\sigma_{\theta}^{\prime}+\sigma_{\zeta}^{\prime}\right)+\sqrt{\left(\sigma_{\theta}^{\prime}-\sigma_{\zeta}^{\prime}\right)^{2}+4 \tau_{\theta \zeta}^{2}}}{2}$

$\sigma_{3}^{\prime}=\frac{\left(\sigma_{\theta}^{\prime}+\sigma_{\zeta}^{\prime}\right)-\sqrt{\left(\sigma_{\theta}^{\prime}-\sigma_{\zeta}^{\prime}\right)^{2}+4 \tau_{\theta \zeta}^{2}}}{2}$

with corresponding eigenvectors

$\vec{\sigma}_{1}^{\prime}=\left(\begin{array}{l}1 \\ 0 \\ 0\end{array}\right)$

$\vec{\sigma}_{2}^{\prime}=\frac{1}{\left(\sigma_{\theta}^{\prime}-\sigma_{2}^{\prime}\right)^{2}+4 \tau_{\theta \zeta}^{2}}\left(\begin{array}{c}0 \\ \tau_{\theta \zeta} \\ -\left(\sigma_{\theta}^{\prime}-\sigma_{2}^{\prime}\right)\end{array}\right)$

$\vec{\sigma}_{3}^{\prime}=\frac{1}{\left(\sigma_{\theta}^{\prime}-\sigma_{2}^{\prime}\right)^{2}+4 \tau_{\theta \zeta}^{2}}\left(\begin{array}{c}0 \\ \tau_{\theta \zeta} \\ -\left(\sigma_{\theta}^{\prime}-\sigma_{3}^{\prime}\right)\end{array}\right)$

Assuming that $\sigma_{3}^{\prime}$ is the smallest, and hence the most tensile principal stress, tensile failure occurs when

$$
\sigma_{3}^{\prime}=\sigma_{t}
$$

where $\sigma_{t}$ is the tensile strength of rock.

Since the minimum principal stresses direction is perpendicular to the fracture trace:

$$
\tan \beta=\frac{\sigma_{3}^{\prime}-\sigma_{\theta}^{\prime}}{\tau_{\theta \zeta}}
$$

Alternatively, since $\sigma_{r}=P_{w}$ is one of the principal stresses, we also get

$$
\tan (2 \beta)=\frac{2 \tau_{\theta \zeta}}{\sigma_{\theta}^{\prime}-\sigma_{\zeta}^{\prime}}
$$

Furthermore 


$$
\frac{\partial \sigma_{3}^{\prime}}{\partial \theta}=0
$$

where

$$
\begin{aligned}
& \frac{\partial \sigma_{3}^{\prime}}{\partial \theta}=\frac{1}{2}\left(\frac{\partial \sigma_{\theta}^{\prime}}{\partial \theta}+\frac{\partial \sigma_{z}^{\prime}}{\partial \theta}\right)-\frac{1}{4}\left(\left(\sigma_{\theta}^{\prime}-\sigma_{\zeta}^{\prime}\right)^{2}+4 \tau_{\theta \zeta}^{2}\right)^{-\frac{1}{2}}\left(2\left(\sigma_{\theta}^{\prime}-\sigma_{\zeta}^{\prime}\right)\left(\frac{\partial \sigma_{\theta}^{\prime}}{\partial \theta}-\frac{\partial \sigma_{\zeta}^{\prime}}{\partial \theta}\right)+8 \tau_{\theta \zeta} \frac{\partial \tau_{\theta \zeta}}{\partial \theta}\right) \\
& \frac{\partial \sigma_{\theta}^{\prime}}{\partial \theta}=4\left(\sigma_{x}-\sigma_{y}\right) \sin (2 \theta)-8 \tau_{x y} \cos (2 \theta) \\
& \frac{\partial \sigma_{\zeta}^{\prime}}{\partial \theta}=4 v\left(\sigma_{x}-\sigma_{y}\right) \sin (2 \theta)-8 v \tau_{x y} \cos (2 \theta) \\
& \frac{\partial \tau_{\theta \zeta}}{\partial \theta}=-2 \tau_{x z} \cos \theta-2 \tau_{y z} \sin \theta
\end{aligned}
$$

For $\sigma_{3}^{\prime}$ to be a minimum,

$$
\frac{\partial^{2} \sigma_{3}^{\prime}}{\partial \theta^{2}}>0
$$

and by solving Eq. (10), the circumferential angle $\theta_{\mathrm{m}}$ where fracture is initiated can be obtained.

It should be noted that $\beta$ depends on the signs of $\tau_{\theta \zeta}$ and $\sigma_{\theta}^{\prime}-\sigma_{\zeta}^{\prime}$ and Table 1 shows how to determine $\beta$ from Eq. (9).

Table 1 Fracture trace angle determination

\begin{tabular}{|c|c|}
\hline$\tau_{\theta \zeta}>0, \sigma_{\theta}^{\prime}<\sigma_{\zeta}^{\prime}$ & $0^{\circ}<\beta<45^{\circ}$ \\
\hline$\tau_{\theta \zeta}<0, \sigma_{\theta}^{\prime}<\sigma_{\zeta}^{\prime}$ & $-45^{\circ}<\beta<0^{\circ}$ \\
\hline$\tau_{\theta \zeta}>0, \sigma_{\theta}^{\prime}>\sigma_{\zeta}^{\prime}$ & $45^{\circ}<\beta<90^{\circ}$ \\
\hline$\tau_{\theta \zeta}<0, \sigma_{\theta}^{\prime}>\sigma_{\zeta}^{\prime}$ & $-90^{\circ}<\beta<-45^{\circ}$ \\
\hline$\tau_{\theta \zeta}=0, \sigma_{\theta}^{\prime}<\sigma_{\zeta}^{\prime}$ & $\beta=0^{\circ}$ \\
\hline$\tau_{\theta \zeta}=0, \sigma_{\theta}^{\prime}>\sigma_{\zeta}^{\prime}$ & $\beta=90^{\circ}$ \\
\hline
\end{tabular}

\section{In situ stresses by testing vertical holes}

In the case of vertical boreholes, the wellbore fluid pressures required to initiate longitudinal tensile hydraulic fractures is (Hubbert and Willis, 1957) 


$$
P_{w} \geq 3 \sigma_{\mathrm{h}}-\sigma_{\mathrm{H}}-P_{0}+\sigma_{t}
$$

where $\sigma_{\mathrm{H}}$ and $\sigma_{\mathrm{h}}$ are the largest and smallest in situ horizontal principal stresses.

As shown in Eq. (3), $\sigma_{\zeta}^{\prime}$ is independent of $P_{w} . \sigma_{\zeta}^{\prime}$ remains constant as $P_{w}$ increases, but $\sigma_{\theta}^{\prime}$ decreases as $P_{w}$ increases. It is obvious that $\sigma_{\theta}^{\prime}$ will always be smaller than $\sigma_{\zeta}^{\prime}$ during hydraulic pressurization and will be the least compressive principal stress. In this case hydraulic fractures are always vertical and $\beta=0$.

The shut-in pressure $\left(P_{s}\right)$ is the pressure needed to keep the fracture barely open, which in this case is $\sigma_{\mathrm{h}}$

$$
\sigma_{\mathrm{h}}=P_{s}
$$

The direction of $\sigma_{\mathrm{h}}$ is normal to vertical hydraulic fracture.

The largest in situ horizontal principal stress is calculated based on Eq. (12) which assumes linear elasticity and that the effect of fracturing on fluid rock infiltration is insignificant. More elaborate pore pressure corrections have been proposed (e.g. Haimson and Fairhurst, 1967) which outline the necessity to better understand coupling effects and their dependency on the local stress state.

\section{In situ stresses by testing inclined holes}

In case of inclined holes, Eq. (3) cannot be solved explicitly, although several attempts have been made to derive in situ stresses from hydraulic fracturing data.

Method of Haimson (1993)

Haimson (1993) considered a special case in which the hole is inclined in the direction of the maximum horizontal stress $\sigma_{\mathrm{H}}$ (wellbore azimuth $\varphi=90^{\circ}$ ). In this case Eq. (3) can be written in terms of the in situ stress

$$
\begin{aligned}
& \sigma_{r}=P_{w} \\
& \sigma_{\theta}=\sigma_{\mathrm{H}} \cos ^{2} \gamma+\sigma_{\mathrm{v}} \sin ^{2} \gamma+\sigma_{\mathrm{h}}-2\left(\sigma_{\mathrm{H}} \cos ^{2} \gamma+\sigma_{\mathrm{v}} \sin ^{2} \gamma-\sigma_{\mathrm{h}}\right) \cos (2 \theta)-P_{w} \\
& \sigma_{\zeta}=\sigma_{\mathrm{H}} \sin ^{2} \gamma+\sigma_{\mathrm{v}} \cos ^{2} \gamma-2 v\left(\sigma_{\mathrm{H}} \cos ^{2} \gamma+\sigma_{\mathrm{v}} \sin ^{2} \gamma-\sigma_{\mathrm{h}}\right) \cos (2 \theta) \\
& \tau_{r \theta}=0 \\
& \tau_{\theta \zeta}=2\left(\sigma_{\mathrm{v}}-\sigma_{\mathrm{H}}\right) \cos \gamma \sin \gamma \sin \theta \\
& \tau_{\zeta r}=0
\end{aligned}
$$


The shear stress $\tau_{\theta \zeta}$ vanishes when $\theta=0$ or $\theta=180^{\circ}$ which implies that $\sigma_{\theta}$ at these points is a principal stress. Neglecting shear stresses, Haimson (1993) suggested using the following equation to obtain $\sigma_{\mathrm{H}}$

$$
\sigma_{\mathrm{H}}=\left(3 \sigma_{\mathrm{h}}-P_{w}-\sigma_{\mathrm{v}} \sin ^{2} \gamma\right) / \cos ^{2} \gamma
$$

Although $\sigma_{\theta}$ becomes a principal stress at $\theta=0$ or $\theta=180^{\circ}$, it is not necessarily the least compressive principal stress compared with other locations around the circumference from $\theta=0$ to $360^{\circ}$. Huang et al. (2010) showed that under normal faulting $\left(\sigma_{\mathrm{v}}>\sigma_{\mathrm{H}}>\sigma_{\mathrm{h}}\right)$ stress condition, the angular position of the fracture $\left(\theta_{\mathrm{m}}\right)$ and the fracture trace angle $(\beta)$ are both zero. However, for reverse faulting $\left(\sigma_{\mathrm{H}}>\sigma_{\mathrm{h}}>\sigma_{\mathrm{v}}\right)$ and strike slip faulting $\left(\sigma_{\mathrm{H}}>\sigma_{\mathrm{v}}>\sigma_{\mathrm{h}}\right)$ stress conditions, $\theta_{\mathrm{m}}$ could be at $90^{\circ}$ when the wellbore inclination is high. In this case, the relation between the breakdown pressure and $\sigma_{\mathrm{H}}$ is nonlinear as indicated by

$$
P_{w}=\sigma_{\mathrm{h}}+\frac{4\left(\sigma_{\mathrm{v}}-\sigma_{\mathrm{H}}\right)^{2} \cos ^{2} \gamma \sin ^{2} \gamma}{\sigma_{\mathrm{H}} \sin ^{2} \gamma+\sigma_{\mathrm{v}} \cos ^{2} \gamma}-3 \sigma_{\mathrm{H}} \cos ^{2} \gamma-3 \sigma_{\mathrm{v}} \sin ^{2} \gamma
$$

\section{Method of Djurhuus and Aadnøy (2003)}

Djurhuus and Aadnøy (2003) presented a theory for determining the in situ stress state from multiple fracturing data and induced fractures from image logs. The position of the fracture on the borehole wall was determined by minimization of the tangential stress $\sigma_{\theta}^{\prime}$, resulting in the equation

$$
\tan (2 \theta)=2 \frac{-\tau_{x z} \tau_{y z}+\tau_{x y}\left(\sigma_{z}-P_{0}\right)}{\tau_{y z}^{2}-\tau_{x z}^{2}+\left(\sigma_{x}-\sigma_{y}\right)\left(\sigma_{z}-P_{0}\right)}
$$

By neglecting the shear stresses, Eq. (17) reduced to

$$
\tan (2 \theta)=\frac{2 \tau_{x y}}{\sigma_{x}-\sigma_{y}}
$$

thus the fracturing position on the borehole wall calculated from Eq. (18) will be either $\theta=0^{\circ}$ or $\theta=90^{\circ}$.

At tensile failure (assuming rock tensile strength is zero):

when, $\theta=0^{\circ}$, and $\sigma_{x}>\sigma_{y}$ 


$$
3 \sigma_{y}-\sigma_{x}-P_{w}-P_{0}=0
$$

and when, $\theta=90^{\circ}$, and $\sigma_{x}<\sigma_{y}$

$$
3 \sigma_{x}-\sigma_{y}-P_{w}-P_{0}=0
$$

After substitution of the stress transformation equations, the above equations becomes

$$
\begin{aligned}
& \sigma_{\mathrm{H}}\left(3 \sin ^{2} \varphi-\cos ^{2} \varphi \cos ^{2} \gamma\right)+\sigma_{\mathrm{h}}\left(3 \cos ^{2} \varphi-\sin ^{2} \varphi \cos ^{2} \gamma\right) \\
& -\sigma_{\mathrm{v}} \sin ^{2} \gamma-P_{w}-P_{0}=0 \text { if } \sigma_{x}>\sigma_{y} \\
& \sigma_{\mathrm{H}}\left(3 \cos ^{2} \varphi \cos ^{2} \gamma-\sin ^{2} \varphi\right)+\sigma_{\mathrm{h}}\left(3 \sin ^{2} \varphi \cos ^{2} \gamma-\cos ^{2} \varphi\right) \\
& +3 \sigma_{\mathrm{v}} \sin ^{2} \gamma-P_{w}-P_{0}=0 \text { if } \sigma_{x}<\sigma_{y}
\end{aligned}
$$

Aadnøy (1990a) redefined Eqs. (21) and (22) in the form

$$
P=a \frac{\sigma_{\mathrm{H}}}{\sigma_{\mathrm{v}}}+b \frac{\sigma_{\mathrm{h}}}{\sigma_{\mathrm{v}}}
$$

and in combination with a number of data sets, the two unknown horizontal in situ stresses $\sigma_{\mathrm{H}}$ and $\sigma_{\mathrm{h}}$ were determined from Eq. (23) using the and least square method. Gjønnes et al. (1998) used a similar approach, but shear stress was included.

Provided $\sigma_{\mathrm{H}}$ and $\sigma_{\mathrm{h}}$ have been obtained, Djurhuus and Aadnøy (2003) further determined $\gamma$ and $\varphi$ from Eqs. (9) and (18) but the back-figured values of $\gamma$ and $\varphi$ were not the same as the originally assumed values.

Method of Okabe et al. (1998)

Okabe et al. (1998) investigated the feasibility of estimating an in situ three-dimensional stress field by using data of drilling-induced tensile fractures observed in a single inclined borehole. Based on Eqs. (9) and (10):

$$
\begin{aligned}
& \theta_{\mathrm{m}}=f\left(\sigma_{x}^{\infty}, \sigma_{y}^{\infty}, \sigma_{z}^{\infty}, \tau_{x y}^{\infty}, \tau_{y z}^{\infty}, \tau_{z x}^{\infty}, \varphi, \gamma, \sigma_{t}, \nu, P_{w}\right) \\
& \beta=g\left(\sigma_{x}^{\infty}, \sigma_{y}^{\infty}, \sigma_{z}^{\infty}, \tau_{x y}^{\infty}, \tau_{y z}^{\infty}, \tau_{z x}^{\infty}, \varphi, \gamma, \sigma_{t}, \nu, P_{w}\right)
\end{aligned}
$$

and knowing $\varphi, \gamma, \sigma_{t}, v$, and $P_{w}$ and three data sets of $\theta_{\mathrm{m}}$ and $\beta$ ( $\varphi$ and $\gamma$ are the borehole azimuth and inclination defined in coordinate system $\left.\left(x^{\infty}, y^{\infty}, z^{\infty}\right)\right), \sigma_{x}^{\infty}, \sigma_{y}^{\infty}, \sigma_{z}^{\infty}, \tau_{x y}^{\infty}, \tau_{y z}^{\infty}, \tau_{z x}^{\infty}$ can be determined from Eqs. (24) and (25) using the least square method. 


\section{Proposed method}

It is assumed that the vertical principal stress $\sigma_{\mathrm{v}}$ is known, together with the borehole inclination $\gamma$. This leaves three unknowns, namely the magnitude of the horizontal principal stresses $\sigma_{\mathrm{H}}$ and $\sigma_{\mathrm{h}}$, and the horizontal in situ stress azimuth with respect to the borehole coordinate system $\varphi$.

Using observed data $P_{w}, \theta_{\mathrm{m}}$ and $\beta$, the in situ stresses $\sigma_{\mathrm{H}}$ and $\sigma_{\mathrm{h}}$ and $\varphi$ can be obtained by solving the simultaneous nonlinear equations (7), (9) and (10). Any conventional numerical nonlinear equation solver (e.g. Newton-Raphson) can be used.

\section{Numerical examples}

\section{North Sea}

Aadnøy (1990a, 1990b) and Djurhuus and Aadnøy (2003) presented inversion results of in-situ stresses for a small offshore field in the North Sea. Nine data sets from three wells covering a depth interval from about one kilometer to three kilometers were analyzed. Data set 2, 5 and 8 were from depth interval $1600 \mathrm{~m}-1800 \mathrm{~m}$ and listed in Table 2. Image analysis was performed for data set 2. The angle where fracture is initiated $\theta_{\mathrm{m}}=43^{\circ}$ and the fracture trace angle $\beta=30^{\circ}$ were obtained.

Table 2 Data set 2, 5 and 8

\begin{tabular}{|c|c|c|c|c|c|}
\hline Data set & Depth & $P_{w}(s g)$ & $P_{0}(s g)$ & $\sigma_{\mathrm{v}}(s g)$ & $\gamma\left(^{\circ}\right)$ \\
\hline 2 & 1880 & 1.84 & 1.39 & 1.82 & 27 \\
\hline 5 & 1812 & 1.78 & 1.25 & 1.82 & 42 \\
\hline 8 & 1607 & 1.71 & 1.05 & 1.78 & 48 \\
\hline
\end{tabular}

Assuming $\varphi=-47^{\circ}$ with data sets 2, 5, 8 and Eq. (23), the following stress ratios were obtained, $\frac{\sigma_{\mathrm{H}}}{\sigma_{\mathrm{v}}}=0.919$ and $\frac{\sigma_{\mathrm{h}}}{\sigma_{\mathrm{v}}}=0.837$.

Djurhuus and Aadnøy (2003) then used these stress ratios to back-figure the inclination of the borehole from Eqs. (9) and (12), leading to $\gamma=36^{\circ}$, which differs from the original value by $9^{\circ}$. This inconsistency may need to be corrected by further calibration.

Using the method proposed in this paper with data set 2 and $\beta=30^{\circ}, \theta_{\mathrm{m}}=43^{\circ}$ from image analysis, the following results were obtained, $\frac{\sigma_{\mathrm{H}}}{\sigma_{\mathrm{v}}}=0.800, \frac{\sigma_{\mathrm{h}}}{\sigma_{\mathrm{v}}}=0.575$ and $\varphi=-44.38^{\circ}$. The 
Matlab program to solve the simultaneous nonlinear equations (7), (9) and (10) is shown in Appendix I.

If $\gamma=36^{\circ}$ is used, the results are changed to $\frac{\sigma_{\mathrm{H}}}{\sigma_{\mathrm{v}}}=0.852, \frac{\sigma_{\mathrm{h}}}{\sigma_{\mathrm{v}}}=0.749 \varphi=-38.09^{\circ}$.

The method in this paper is based on the complete stress solution and uses a single data set including data from image analysis. Results by Djurhuus and Aadnøy (2003) are based on an incomplete stress solution and require at least two data sets. If the fracture trace angle $\beta$ is available, the inversion method proposed in this paper is to be preferred, since it will deliver a consistent stress prediction based on classical mechanics.

\section{Kuparuk River field in Alaska}

Numerous studies addressing the problems of initiation and propagation of fractures from deviated wells have been conducted. Yew et al. (1989) demonstrate that an individual fracture starts in a tensile zone at some angle with the well axis (openhole). The tensile zone and the fracture angle are determined from the plane strain condition given by the Kirsch solution (with superposition of the axial stress) and the Mohr's circle envelope. This criterion is extended to the initiation of several fractures by assuming that the interval between two perforations behaves like an openhole and that adjacent starter fractures link up tip to tip yielding a zigzag fracture trace at the wellbore wall. Yew's analysis was used with some success by Pearson et al. (1992) in the design of perforation spacing for offshore wells in the Kuparuk field of Alaska. The Kuparuk River field, one of the largest oil field in the U.S., is located in the Alaskan Arctic and covers about 115,000 acres. Twenty six wells were drilled in site $2 \mathrm{~K}$ in the latter half of 1989 and early 1990. The in situ stress had previously been found to be $\sigma_{\mathrm{v}}=6200(\mathrm{psi}), \sigma_{\mathrm{H}}=5803(\mathrm{psi})$ and $\sigma_{\mathrm{h}}=4538$ (psi). The completion design was conducted by Pearson et al. (1992) to determine perforation requirements (minimum spacing and orientation). Table 3 shows the data obtained from either laboratory or field measurements. Other parameters are $P_{0}=0, \sigma_{t}=500$ (psi) and $v=0.2$.

The input parameters shown in Table $3\left(\gamma, P_{w}, \theta_{\mathrm{m}}\right.$ and $\left.\beta\right)$ and $\sigma_{\mathrm{v}}=6200(\mathrm{psi}), P_{0}=0$, $\sigma_{t}=500$ (psi) and $v=0.2$ were used with the Matlab program shown in Appendix I to compute he inversion results listed in Table 4 by solving the simultaneous nonlinear equations (7), (9) and (10) to obtain $\varphi, \sigma_{\mathrm{h}}$ and $\sigma_{\mathrm{H}}$. The results are in generally excellent agreement with the observed field values $\sigma_{\mathrm{H}}=5803(\mathrm{psi}), \sigma_{\mathrm{h}}=4538(\mathrm{psi})$, and $\varphi$ shown in Table 3 . The only exception to the agreement was observed in the calculated value of $\varphi$ corresponding to data set 3. 
Table 3 Data from either laboratory or field measurements at drill site $2 \mathrm{~K}$

\begin{tabular}{|c|c|c|c|c|c|}
\hline Data set & $\varphi\left(^{\circ}\right)$ & $\gamma\left(^{\circ}\right)$ & $P_{w}$ (psi) & $\theta_{\mathrm{m}}\left(^{\circ}\right)$ & $\beta\left(^{\circ}\right)$ \\
\hline 1 & 30 & 15 & 8415 & 57.8 & 6.6 \\
\hline 2 & 30 & 30 & 8850 & 49.9 & 11.5 \\
\hline 3 & 30 & 60 & 9426 & 17.6 & 10.6 \\
\hline 4 & 60 & 15 & 8260 & 28.0 & 3.7 \\
\hline 5 & 60 & 30 & 8261 & 22.6 & 6.3 \\
\hline 6 & 60 & 60 & 8070 & 9.6 & 7.6 \\
\hline 7 & 90 & 15 & 8187 & 0 & 0 \\
\hline 8 & 90 & 30 & 8017 & 0 & 0 \\
\hline 9 & 90 & 60 & 7552 & 0 & 0 \\
\hline
\end{tabular}

Table 4 Inversion results by the method proposed in this paper

\begin{tabular}{|c|c|c|c|}
\hline Data set & $\varphi\left(^{\circ}\right)$ & $\sigma_{\mathrm{h}}(\mathrm{psi})$ & $\sigma_{\mathrm{H}}(\mathrm{psi})$ \\
\hline 1 & 30.1 & 4767 & 5515 \\
\hline 2 & 26.1 & 4712 & 5286 \\
\hline 3 & 6.2 & 4114 & 5256 \\
\hline 4 & 60.4 & 4792 & 5632 \\
\hline 5 & 62.0 & 4812 & 5714 \\
\hline 6 & 57.2 & 4720 & 5364 \\
\hline 7 & 90.0 & 4205 & 4342 \\
\hline 8 & 90.0 & 4152 & 4394 \\
\hline 9 & 83.5 & 5029 & 5044 \\
\hline
\end{tabular}

\section{Concluding Remarks}

A new method for determination of the in situ stress state from hydraulic fracturing data from inclined boreholes is presented and shown to give good agreement with published field observation data. The solution is based on classical mechanics and uses a single data set which includes breakdown pressure together with the fracture angular position and trace angle. The method proposed in this paper avoids the inconsistencies that can occur with existing incomplete methods. 


\section{Notation}

$\sigma_{\mathrm{v}} \quad$ vertical in situ stress

$\sigma_{\mathrm{H}} \quad$ major horizontal in situ stress

$\sigma_{\mathrm{h}} \quad$ minor horizontal in situ stress

$\gamma \quad$ wellbore inclination

$\varphi \quad$ azimuth, angle between $\sigma_{\mathrm{H}}$ and wellbore $X$ axis

$\left\{\begin{array}{l}\sigma_{1} \\ \sigma_{2} \\ \sigma_{3}\end{array}\right\}$ principal stresses

$\left\{\begin{array}{c}\sigma_{x} \\ \sigma_{y} \\ \sigma_{z} \\ \tau_{x y} \\ \tau_{y z} \\ \tau_{z x}\end{array}\right\}$

far field in situ stress transformed to wellbore coordinate system

$\left\{\begin{array}{c}\sigma_{x}^{\infty} \\ \sigma_{y}^{\infty} \\ \sigma_{z}^{\infty} \\ \tau_{x y}^{\infty} \\ \tau_{y z}^{\infty} \\ \tau_{x z}^{\infty}\end{array}\right\}$

far field in situ stress

$\left\{\begin{array}{c}\sigma_{r} \\ \sigma_{\theta} \\ \sigma_{\zeta} \\ \tau_{r \theta} \\ \tau_{\theta \zeta} \\ \tau_{\zeta r}\end{array}\right\}$

stress at the wellbore wall

a wellbore radius 
$P_{0} \quad$ pore pressure.

$P_{s} \quad$ shut-in pressure

$P_{w} \quad$ wellbore pressure

$r \quad$ radial distance from the wellbore center

$\sigma_{t} \quad$ tensile strength of rock.

$\beta \quad$ fracture tracer angle

$\theta_{\mathrm{m}} \quad$ angle where fracture is initiated

$v \quad$ Poisson 's ratio

Primes indicate effective stress.

\section{Acknowledgement}

The authors wish to acknowledge the support of NSF grant CMMI-0970122 on "GOALI: Probabilistic Geomechanical Analysis in the Exploitation of Unconventional Resources."

\section{References}

Aadnøy, B.S., 1990a. Inversion technique to determine the in-situ stress field from fracturing data. Journal of Petroleum Science and Engineering 4, 127- 141.

Aadnøy, B.S., 1990b. In-situ stress directions from borehole fracture traces. Journal of Petroleum Science and Engineering 4, 143- 153.

Aadnøy, B.S. and J.S. Bell (1998): Classification of drill-induce fractures and their relationship to insitu stress directions. - Log Analyst, 39, 27-42.

Barton, C.A., Moos, D., Peska, P., and Zoback, M.D., 1997. Utilizing wellbore image data to determine the complete stress tensor: application to permeability anisotropy and wellbore stability. Log Anal., 38:21-33.

Bradley, W. B., Failure of Inclined Wellbores. J. Energy Res. Tech., Trans. AIME, 102, 232-239 (1979). 
Chen, Z.M. and Economides, M.J.: "Effect of Near Wellbore Fracture Geometry on Fracture Execution and Post-Treatment Well Production of Deviated and Horizontal Wells," SPEPF (August 1999) 177-186.

Daneshy, A. A. (1971), True and apparent direction of hydraulic fractures, Soc. Petrol. Eng., paper no. SPE3226.

Djurhuus, J., and B.S. Aadnoy. 2003. In situ stress state from inversion of fracturing data from oil wells and wellbore image logs. J. Petrol. Sci. Eng. 38: 121-130.

El-Rabaa, W., 1989. Experimental study of hydraulic fracture geometry initiated from horizontal wells. In: SPE 19720. Ann. Technical Conf., San Antonio, TX, Oct. 8-11.

Fairhurst, C., (1964). Measurement of in situ rock stresses, with particular reference to hydraulic fracturing. Felsmech. Ingenieurgeol. 3-4, pp129-147.

Fairhurst, C., (1968). Methods of determining in-situ rock stresses at great depth. TRI-68 Missouri River Div., Corps of Engineer.

Gjønes M, Cruz AMGL, Horsrud P, Holt RM. Leak-off tests for horizontal stress determination? J Pet Sci Eng 1998; 20:63-71.

Haimson B.C., (1993). The hydraulic fracturing method of stress measurement: theory and practice. In: Hudson JA, editor-in chief, Comprehensive rock engineering, vol. 3; p. 395-412.

Haimson B.E., Cornet F.H. (2003). ISRM suggested methods for rock stress estimation-Part 3: hydraulic fracturing (HF) and/or hydraulic testing of pre-existing fractures (HTPF). Int J Rock Mech Min Sci, 40:1011-20.

Haimson, B. C. and C. Fairhurst, Initiation and Extension of Hydraulic Fracture in Rocks, Soc. Petr. Engrs. J., Sept., 310-318 (1967).

Huang, J., Griffiths, D.V., and Wong, S.W. (2010) "Hydraulic fracture: initiation pressure, location and orentation." Under review by International Journal for Numerical and Analytical Methods in Geomechanics.

Hubbert, M.K., Willis, D.G., (1957). Mechanics of hydraulic fracturing. Trans. AIME 210, 153166.

Kirsch, 1898, Die Theorie der Elastizität und die Bedürfnisse der Festigkeitslehre. Zeitschrift des Vereines deutscher Ingenieure, 42, 797-807.

Kuriyagawa, M., H. Kobayashi, I. Matsunaga, T. Yamaguchi \& K. Hibiya 1989. Application of hydraulicf racturingt o three-dimensionainl situ stress measurement. lnt. J. Rock Mech. \& Min. Sci. 26: 587-593.

Okabe, T., Hayashi, K., Shinohara, N., Takasugi, S., 1998. Inversion of drilling-induced tensile fracture data obtained from a single inclined wellbore. International Journal of Rock Mechanics and Mining Sciences 35 (6), 747- 758. 
Peška, P. \& M.D. Zoback 1995. Compressive tensile failure of inclined wellbores and determination of in situ stress and rock strength. J. GeophysR. es. 100: 12791-12811.

Pearson, C.M., Bond, A.J., Eck, M.G., Schmidt, J.H., 1992. Results of stress-oriented and aligned perforating in fracturing deviated wells. J. Pet. Technol., 10-18, Jan. 10-18.

Yew, C.H. (1997). Mechanics of Hydraulic Fracturing, Gulf Professional Publishing.

Yew, C.H., Schmith, J.R. and Li, Y.: "On Fracture Design of Deviated Wells," paper SPE 19722 presented at the 1989 SPE Annual Technical Conference and Exhibition, San Antonio, Oct. 8-11.

\section{Appendix I Matlab program of stress inversion}

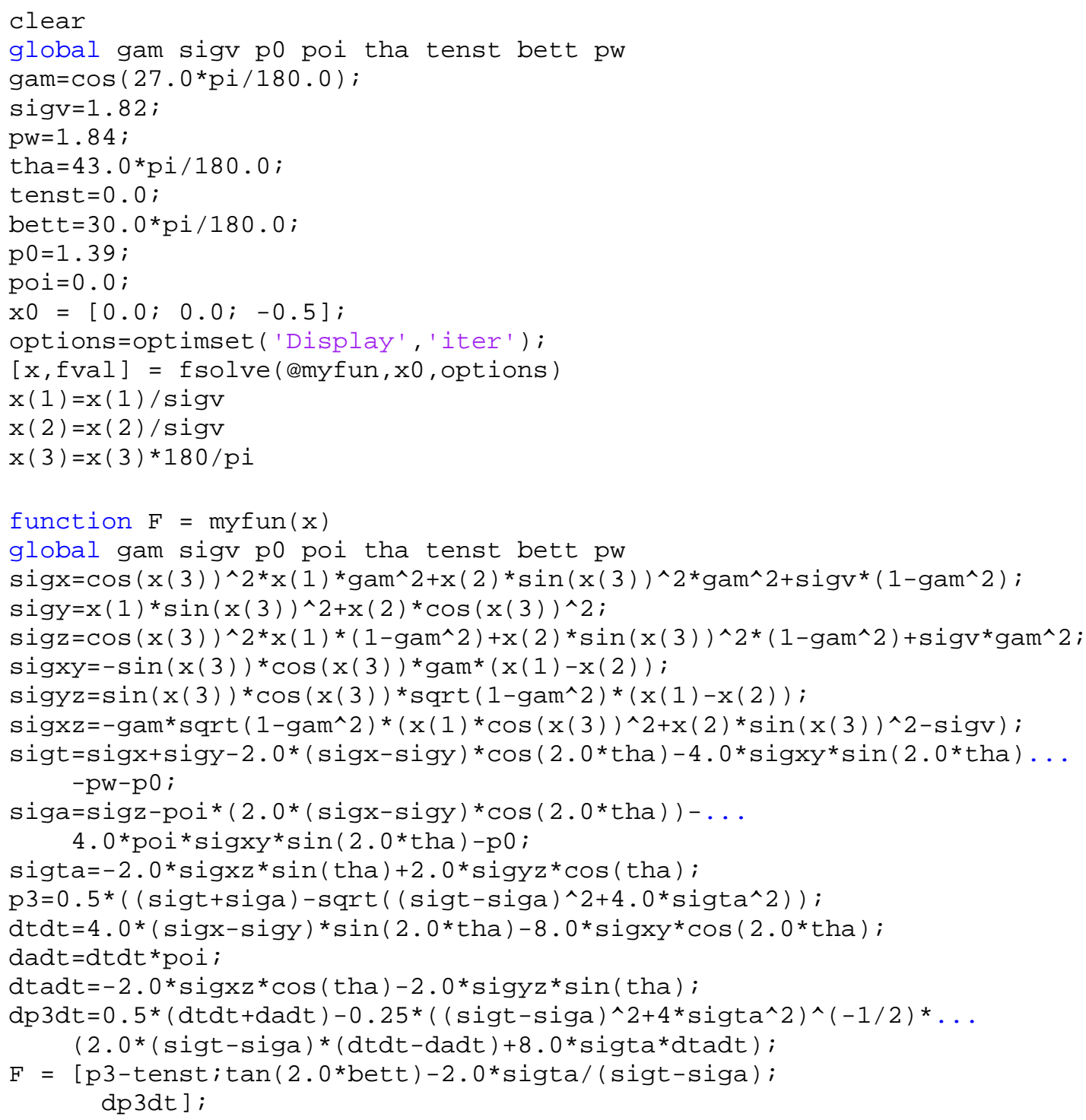

